\title{
PDE APPROACH TO INVARIANT AND GIBBS MEASURES WITH APPLICATIONS
}

\author{
MICHAEL RÖCKNER \\ Fakultät für Mathematik \\ Universität Bielefeld \\ D-33615 Bielefeld (Germany) \\ Email: roeckner@mathematik.uni-bielefeld.de
}

\begin{abstract}
In this paper we give a pedagogical account of the PDE approach to invariant and Gibbs measures in finite and infinite dimensions. As an application we describe some recent new results on the classical problem whether "invariance implies Gibbsian" and illustrate how they apply to a well-studied lattice model from statistical mechanics with non-compact single spin spaces.
\end{abstract}

AMS 1991 Subject classification:

Primary: 58G32, 58B99, 82B21.

Secondary: 53C21, 53C80, 58G03, 60J60, 82B05.

Keywords and phrases: invariant measures, diffusions on manifolds, elliptic equations for measures, Lyapunov functions, Gibbs distributions, logarithmic gradients

\section{Introduction}

This paper is an extended version of my talk given at the symposium in Tübingen. The main purpose is to give a concise pedagogical account on the PDE (= partial differential equation) approach to invariant and Gibbs measures, developed in a series of papers during the last few years by S. Albeverio, V.I. Bogachev, Y.G. Kondratiev, T. Pasurek, F.Y. Wang and the author (cf. e.g. $A K R 97 a, A K R 97 b, A K R T 00, B R W 01, B R 01, A K P R 04$ ), and describe a recent new application to the classical problem whether any invariant measure is Gibbsian (cf. ${ }^{B R W 02}$ ). The latter will be done in more detail, but the main result will be only precisely formulated, without recalling the proof from $B R W 02$. Instead, we shall present an application to an intensively studied model from statistical mechanics (see e.g. ${ }^{B H} K 82$ ). 


\section{The PDE-approach to invariant and Gibbs measures}

\subsection{The finite dimensional case}

Consider the $d$-dimensional Euclidean space $\mathbb{R}^{d}$ (or more general a $d$ dimensional manifold $M^{d}$ ) and a Borel measurable function $E: \mathbb{R}^{d} \rightarrow$ $(-\infty,+\infty]$ such that $e^{-E} \in L^{1}\left(\mathbb{R}^{d}, d x\right)$ where $d x$ denotes Lebesgue measure. For a better understanding of the following one should think of $E(x)$ as the "energy" of the "configuration" $x=\left(x_{i}\right)_{1 \leq i \leq d} \in \mathbb{R}^{d}$. Let us assume for simplicity that $E \in C^{1}\left(\mathbb{R}^{d}\right)$. For a probability measure $\mu$ on $\mathbb{R}^{d}$ consider the following assertions:

(1) $\mu$ is a Gibbs measure (with energy $E$ ), i.e.

$$
\mu(d x)=\left(\int e^{-E(x)} d x\right)^{-1} e^{-E(x)} d x .
$$

(2) $\mu$ satisfies the following first order PDE:

$$
\partial_{i} \mu=-\partial_{i} E \cdot \mu \quad \forall 1 \leq i \leq d
$$

(where $\partial_{i}:=\frac{\partial}{\partial x_{i}}$ and $\partial_{i} \mu$ denotes the distributional derivative of $\mu$ ).

(3) Setting $Z:=\left(Z_{i}\right)_{1 \leq i \leq d}, Z_{i}:=\partial_{i} E$ and $L_{Z}:=\sum_{i=1}^{d}\left(\partial_{i}^{2}-Z_{i} \partial_{i}\right)$, then $\mu$ symmetrizes $L_{Z}$ with domain $C_{0}^{2}\left(\mathbb{R}^{d}\right)$, i.e.

$$
\int L_{Z} u v d \mu=\int u L_{Z} v d \mu \quad \forall u, v \in C_{0}^{2}\left(\mathbb{R}^{d}\right) .
$$

(4) $\mu$ is $L_{Z}$-infinitesimally invariant, i.e.

$$
\int L_{Z} u d \mu=0 \quad \forall u \in C_{0}^{2}\left(\mathbb{R}^{d}\right)
$$

or shortly,

$$
L_{Z}^{*} \mu=0
$$

(so $\mu$ satisfies a second order PDE).

Then $(1) \Leftrightarrow(2) \Leftrightarrow(3) \Rightarrow(4)$.

Under our present smoothness assumptions, i.e. $E \in C^{1}\left(\mathbb{R}^{d}\right)$, the implications $(1) \Rightarrow(2) \Rightarrow(3)$ are pretty much obvious. For $(2) \Rightarrow(1)$ a regularity result to ensure the existence of a sufficiently regular RadonNikodym derivative with respect to $d x$ for any $\mu$ satisfying (2) is necessary. For details on the latter in a much more general case, namely where merely $e^{-\frac{1}{2} E} \in H_{\text {loc }}^{1,2}\left(\mathbb{R}^{d}, d x\right)$ (= local Sobolev space of order 1 in $L_{\text {loc }}^{2}\left(\mathbb{R}^{d}, d x\right)$ ) and $e^{-\frac{1}{2} E}>0 d x$-a.e. is assumed, we refer e.g. to Proposition 1.5 and its 
proof in $A R Z 93$ (see also Remark 2.1 below). For $(3) \Rightarrow(2)$ we refer e.g. to Lemma 1 in $B R 03$.

The equivalence of (1) and (2) is of great importance since as we shall see in the next section it generalizes to infinite dimensions, so gives the possibility to study Gibbs measures by PDE-methods.

Concerning the relation between (3) and (4) by choosing $v_{n} \in C_{0}^{2}\left(\mathbb{R}^{d}\right)$ with $v_{n} \equiv 1$ on a ball of radius $n$ and letting $n \rightarrow \infty$ we obviously deduce (4). So, we have "Gibbsian $\Rightarrow$ infinitesimally invariance". The converse in infinite dimension is a famous conjecture of Gibbs (originally formulated for so-called Hamiltonian dynamics).

Under our present regularity assumption on $E$, i.e. $E \in C^{1}\left(\mathbb{R}^{d}\right)$, in this finite dimensional case the converse is also true. This follows from two highly non-trivial general results from Corollary 2.3 (see in particular also Remark 2.4.(i)) in ${ }^{S t a 99}$ and Theorem 3.1 in ${ }^{B R S 00}$ (see also Theorem 4.1 in $B R S 02$ ) which imply that the PDE in (4) has a unique solution, which since $\mu$ in (1) is a solution, is therefore Gibbsian.

If one relaxes the assumptions on $E$ the situation becomes much more complicated. We summarize this in the following remark.

Remark 2.1. For $E: \mathbb{R}^{d} \rightarrow(-\infty, \infty]$ let us only assume the already mentioned condition

$$
e^{-\frac{1}{2} E} \in H_{\mathrm{loc}}^{1,2}\left(\mathbb{R}^{d}, d x\right) \text { and } e^{-\frac{1}{2} E}>0 d x \text {-a.e. }
$$

Setting $\rho:=e^{-E}\left(\in H_{\text {loc }}^{1,1}\left(\mathbb{R}^{d}, d x\right)\right)$ we can reformulate $(2)$ as follows:

(2) $\mu$ satisfies the following first order PDE

$$
\partial_{i} \mu=\left(\frac{\partial_{i} \rho}{\rho}\right)^{\sim} \cdot \mu \quad \text { and } \quad\left(\frac{\partial_{i} \rho}{\rho}\right)^{\sim} \in L_{\mathrm{loc}}^{2}\left(\mathbb{R}^{d}, \mu\right) \quad \forall 1 \leq i \leq d,
$$

for some (Borel) $d x$-version $\left(\frac{\partial_{i} \rho}{\rho}\right)^{\sim}$ of $\frac{\partial_{i} \rho}{\rho}$.

Here we set as usual $\frac{\partial_{i} \rho}{\rho}:=0$ on $\{\rho=0\}$. In this case as mentioned above always $\mu \ll d x$, so the superfix " " can be dropped a posteriori. Note that in general $\frac{\partial_{i} \rho}{\rho} \notin L_{\text {loc }}^{1}\left(\mathbb{R}^{d}, d x\right)$, so even if $E \in L_{\text {loc }}^{1}\left(\mathbb{R}^{d}, d x\right)$, in general $\frac{\partial_{i} \rho}{\rho} \neq \partial_{i} E$, since the right hand side is always a Schwartz distribution. Then we still have

$$
(1) \Rightarrow(\overline{2}) \Leftrightarrow(\overline{3}) \Rightarrow(\overline{4}) \text {. }
$$

Here we take $Z_{i}:=\left(\frac{\partial_{i} \rho}{\rho}\right)^{\sim}$ and $(\overline{3}),(\overline{4})$ are just conditions $(3)$, (4) respectively, augmented by the condition $\left(\frac{\partial_{i} \rho}{\rho}\right)^{\sim} \in L_{\mathrm{loc}}^{2}\left(\mathbb{R}^{d}, \mu\right), 1 \leq i \leq d$. Since 
by Theorem 1 in ${ }^{B K R} R 7$ any solution of $L^{*} \mu=0$ is absolutely continuous with respect to $d x$, we can again drop " $\sim$ " a posteriori.

Under condition (2.1) the PDE in $(\overline{2})$ can have infinitely many solutions (cf. Example 6.1 in ${ }^{B R 95}$ and also Remark 3.6(ii) in ${ }^{A K R 97 b}$ ) even for $d=1$. So, $(\overline{2}) \nRightarrow(1)$. If, however, in addition, to $(2.1)$ also $\frac{|\nabla \rho|}{\rho} \in L_{\text {loc }}^{1}\left(\mathbb{R}^{d}, d x\right)$, then $\mu$ from (1) is the unique solution of the PDE in ( $\overline{2})$ (cf. Theorem 6.2 in ${ }^{B R 95}$ and Theorem 1.8(ii) in $\left.{ }^{A B R 99}\right)$. In addition, $\frac{\partial_{i} \rho}{\rho}=\partial_{i} E, 1 \leq i \leq d$, (cf. Lemma 6.4 in ${ }^{B R 95}$ ). On the other hand, even if $\frac{|\nabla \rho|}{\rho} \in L_{\mathrm{loc}}^{1}\left(\mathbb{R}^{d}, d x\right)$, it appears to be unknown whether $(\overline{4}) \Rightarrow(\overline{3})$. But also no counterexample seems to be known. For conditions so that $(\overline{4}) \Rightarrow(\overline{3})$ in the present situaton we refer to Theorem 1.8(i) in ${ }^{A B R 99}$ for the most general result we are aware of that does not require higher integrability of $\frac{|\nabla \rho|}{\rho}$. If one assumes that $\frac{|\nabla \rho|}{\rho} \in L_{\text {loc }}^{d+\varepsilon}\left(\mathbb{R}^{d}, d x\right), \varepsilon>0$, then by the same results from Sta99, $B R S 00, \stackrel{\rho}{B R S 02}$ mentioned above the PDE in $(\overline{4})$ has a unique solution (among probability measures), so $(\overline{4}) \Rightarrow(1)$, hence $(1) \Leftrightarrow(\overline{2}) \Leftrightarrow(\overline{3}) \Leftrightarrow(\overline{4})$ in this case.

If we replace $\mathbb{R}^{d}$ by a suitable manifold, then there are easy examples even for smooth $Z$ where $(4) \nRightarrow(3)$.

Example 2.1. (cf. Remark 2.5(ii) ${ }^{B R W 02}$ ) Let $M$ be a connected complete Riemannian manifold with infinite volume measure $\lambda_{M}$, such that there exists a positive harmonic function $h$, integrable with respect to the volume measure $\lambda_{M}$ (cf. ${ }^{C h u 83},{ }^{L S 84}$ for existence). Choose the vector field $Z$ to be identically equal to zero, so $L_{Z}=\Delta$ (= Laplacian on $M$ ). Define

$$
\mu:=\left(\int h d \lambda_{M}\right)^{-1} h \cdot \lambda_{M}
$$

Then for $u \in C_{0}^{2}(M)$

$$
\int L_{Z} u d \mu=\int \Delta u h d \lambda_{M}=\int u \Delta h d \lambda_{M}=0
$$


since $\Delta h=0$. But for $u, v \in C_{0}^{2}(M)$

$$
\begin{aligned}
& \int L_{Z} u v d \mu \\
& \quad=\int u \Delta v h d \lambda_{M}+\int u v \Delta h d \lambda_{M}+2 \int u\langle\nabla v, \nabla h\rangle d \lambda_{M} \\
& \quad=\int u L_{Z} v d \mu+2 \int u\langle\nabla v, \nabla h\rangle d \lambda_{M} \\
& \quad \neq \int u L_{Z} v d \mu
\end{aligned}
$$

since $h$ cannot be constant because $\lambda_{M}(M)=\infty$. Therefore, $\mu$ is infinitesimally invariant for $L_{Z}$, but not symmetrizing, hence not Gibbsian.

In infinite dimensions much less is known about when or not (4) implies (3). We have included the quite detailed discussion in Remark 2.1 since it displays a typical characteristics in comparing finite and infinite dimensional analysis. Difficulties in the analysis of PDE or differential operators in infinite dimensions are reflected in part in finite dimensions if the coefficients become singular.

The last remark of this subsection concerns the relation between "infinitesimal invariance" and "invariance". We refer to Subsect. 2.5 in BRS00 and Sections 3 and 4 in ${ }^{B R S 02}$ for more details.

Remark 2.2. Assume again that (2.1) holds and that $\mu$ is as in ( $\overline{4}$ ) (cf. Remark 2.1). Set $L:=L_{Z}$. Suppose there exists a closed extension $\left(\hat{L}^{\mu}, D\left(\hat{L}^{\mu}\right)\right)$ of the operator $\left(L, C_{0}^{2}\left(\mathbb{R}^{d}\right)\right)$ on $L^{1}\left(\mathbb{R}^{d}, \mu\right)$ which generates a $C_{0}$-semigroup $\left(T_{t}^{\mu}\right)_{t \geq 0}=\left(e^{t \hat{L}^{\mu}}\right)_{t \geq 0}$ on $L^{1}\left(\mathbb{R}^{d}, \mu\right)$. If $\mu$ is the special measure in (1), then it follows by Corollary 2.3 and Remark 2.4.(ii) in Sta99 that $\left(\hat{L}^{\mu}, D\left(\hat{L}^{\mu}\right)\right)$ must be the closure of $\left(L, C_{0}^{2}\left(\mathbb{R}^{d}\right)\right)$ and this closure really generates a $C_{0}$-semigroup on $L^{1}\left(\mathbb{R}^{d}, \mu\right)$. In this case a simple consideration implies that

$$
\int T_{t}^{\mu} f d \mu=\int f d \mu \text { for all } t>0, f \in L^{1}\left(\mathbb{R}^{d}, \mu\right),
$$

i.e. $\mu$ is $\left(T_{t}^{\mu}\right)_{t \geq 0}$-invariant. For a general $\mu$ satisfying $(\overline{4})$, however, the mere existence of $\left(T_{t}^{\mu}\right)_{t>0}=\left(e^{\hat{L}^{\mu}}\right)_{t \geq 0}$ for a suitable closed extension $\hat{L}^{\mu}$ of $L$ is unknown and, if it exists, it might not be the closure of $L$. So, it is unclear whether $\mu$ will be its invariant measure in the sense of (2.2). On the other hand, if $\left(T_{t}^{\mu}\right)_{t>0}$ exists and satisfies (2.2), by differentiating at $t=0$, we deduce from (2.2) that $\mu$ satisfies $L^{*} \mu=0$. So, even in finite 
dimensions "infinitesimally invariance" seems to be a more general notion than "invariance".

\subsection{The infinite dimensional case}

Consider now the $d$-dimensional lattice $\mathbb{R}^{\mathbb{Z}^{m}}, m \in \mathbb{N}$, instead of $\mathbb{R}^{d}$. (Again we could also consider a product $\prod_{i \in \mathbb{Z}^{d}} M_{i}$ of finite dimensional Riemannian manifolds, cf. ${ }^{B R W 02}$ for details). We are going to restrict the class of "energy"-functionals $E: \mathbb{R}^{\mathbb{Z}^{m}} \rightarrow(-\infty, \infty]$ a bit, with applications to statistical mechanics in mind. So, let

$$
E(x):=\sum_{\substack{\Lambda \subset \mathbb{Z}^{m} \\|\Lambda|<\infty}} U_{\Lambda}\left(x_{\Lambda}\right), \quad x=\left(x_{i}\right)_{i \in \mathbb{R}^{\mathbb{Z}^{m}}}
$$

where $|\Lambda|$ denotes the cardinality of $\Lambda, x_{\Lambda}:=\left(x_{i}\right)_{i \in \Lambda}$, and $U_{\Lambda}: \mathbb{R}^{\Lambda} \rightarrow$ $(-\infty, \infty]$ is Borel-measurable. Of course, (2.3) is purely informal, because this sum almost never converges. $U_{\Lambda}(x)$ is called the "potential of the configuration $x$ in $\Lambda$ " and as before $E(x)$ is the "energy of the configuration $x=\left(x_{i}\right)_{i \in \mathbb{R}^{\mathbb{Z}^{m}}}$ ". Similarly as in finite dimensions one then defines corresponding Gibbs measures $\mu$ on $\mathbb{R}^{\mathbb{Z}^{m}}$ (equipped with the product of the Borel $\sigma$-algebras on $\mathbb{R}$ ) as

(1)' $\mu$ is a Gibbs measure (with energy $E$ ), i.e.

$$
\mu(d x)="\left(\int e^{-E(x)} \prod_{i \in \mathbb{Z}^{m}} d x_{i}\right)^{-1} e^{-E(x)} \prod_{i \in \mathbb{Z}^{m}} d x_{i}, \quad x=\left(x_{i}\right)_{i \in \mathbb{Z}^{m}},
$$

with $d x_{i}:=$ Lebesgue measure on $\mathbb{R}^{1}$.

Of course, also (1)' is purely informal, since an infinite product of Lebesgue measures does not exist in a suitable sense and, as said before, $E(x)$ is not well-defined. But it turns out (and has been well-known for many years) that the expression in the right hand side of the equality in (1)' can be given sense. In general, however, the correspondingly defined measure is not unique. One reason is e.g. that if one defines the right hand side as a limit along a "localizing sequence" $\Lambda_{n} \nearrow \mathbb{Z}^{m}, n \rightarrow \infty$, with $\Lambda_{n}$ finite, the limit might depend on the sequence. Furthermore, "localizing" implies that one has to fix "boundary conditions" outside every $\Lambda_{n}$, and different choices of these might also lead to different limits. A precise definition of a Gibbs measure taking all these issues into account requires the notion of a "local specification" and then a Gibbs measure can be defined by 
determining its conditional probabilities on $\mathbb{R}^{\Lambda}$ for each finite $\Lambda \subset \mathbb{Z}^{m}$ by this specification fixing the configuration outside $\Lambda$, i.e. the measure satisfies the DLR (= Dobrushin-Lanford-Ruelle) equations. We refer e.g. to the exposition in Geo88 for details, since we do not need this below, since this rigorous version of (1)' is as in finite dimensions equivalent to the following, infinite dimensional analogue (2)' of (2) which is rigorous. This equivalence has been proved in $A K R 97 a, A K R 97 b, A K R T 00$ in various frameworks:

(2)' $\mu$ satisfies the following first order PDE (in infinitely many variables):

$$
\partial_{i} \mu=\left(-\sum_{\substack{\Lambda: i \in \Lambda \\ \Lambda \subset \mathbb{Z}^{m},|\Lambda|<\infty}} \partial_{i} U_{\Lambda}\right) \cdot \mu \quad \forall i \in \mathbb{Z}^{m} .
$$

Precise assumptions for "DLR-version of $(1)^{\prime} \Leftrightarrow(2)^{\prime}$ " to hold are e.g.

$$
U_{\Lambda} \in L_{\mathrm{loc}}^{\infty}\left(\mathbb{R}^{\Lambda}, \prod_{i \in \Lambda} d x_{i}\right) \cap H_{\mathrm{loc}}^{1,2}\left(\mathbb{R}^{\Lambda}, \prod_{i \in \Lambda} d x_{i}\right)
$$

for all finite $\Lambda \subset \mathbb{Z}^{m}$ and for some $R>0$ and

$$
U_{\Lambda} \equiv 0 \quad \text { if } \operatorname{diam} \Lambda>R
$$

("finite range interaction").

To define the distributional derivative $\partial_{i} \mu$ in (2)' we need a test function space. As usual in infinite dimensions we take for $\ell \in \mathbb{N} \cup\{\infty\}$

$$
\begin{aligned}
\mathcal{F} C_{b}^{\ell}:=\left\{u: \mathbb{R}^{\mathbb{Z}^{m}} \rightarrow \mathbb{R} \mid\right. & \exists \text { finite } \Lambda \subset \mathbb{Z}^{m} \text { and } g \in C_{b}^{\ell}\left(\mathbb{R}^{\Lambda}\right) \\
& \text { such that } \left.u(x)=g\left(x_{\Lambda}\right) \text { for all } x \in \mathbb{R}^{\mathbb{Z}^{m}}\right\} .
\end{aligned}
$$

Then a probability measure $\mu$ on $\mathbb{R}^{\mathbb{Z}^{m}}$ satisfies the PDE in (2)' if

$$
\int \partial_{i} u d \mu=\int u Z_{i} d \mu \quad \forall u \in \mathcal{F} C_{b}^{1}, i \in \mathbb{Z}^{m},
$$

where

$$
Z_{i}:=\sum_{\substack{\Lambda: i \in \Lambda \\ \Lambda \subset \mathbb{Z}^{m}, \Lambda \text { finite }}} \partial_{i} U_{\Lambda}, \quad i \in \mathbb{Z}^{m} .
$$

Note that by the finite range condition the sum in (2.8) has only finitely many non-zero summands, so $Z_{i}$ is well-definied. As in the preceding subsection we now consider two further assertions about a probability measure $\mu$ on $\mathbb{R}^{\mathbb{Z}^{m}}$ : 
(3)' Setting $Z:=\left(Z_{i}\right)_{i \in \mathbb{Z}^{m}}, Z_{i}$ as in (2.8), and $L_{Z}:=\sum_{i=1}^{\infty}\left(\partial_{i}^{2}-Z_{i} \partial_{i}\right)$, then $\mu$ symmetrizes $L_{Z}$ with domain $\mathcal{F} C_{b}^{2}$, i.e.

$$
\int L_{Z} u v d \mu=\int u L_{Z} v d \mu \quad \forall u, v \in \mathcal{F} C_{b}^{2}
$$

(4)' $\mu$ is $L_{Z}$-infinitesimally invariant, i.e.

$$
\int L_{Z} u d \mu=0 \quad \forall u \in \mathcal{F} C_{b}^{2}
$$

or shortly,

$$
L_{Z}^{*} \mu=0
$$

(so $\mu$ satisfies a second order PDE in infinitely many variables).

We emphasize that since $u$ (and $v$ ) are in $\mathcal{F} C_{b}^{2}$ the sum in the definition of $L_{Z} u$ has again only finitely many non-zero summands. So, all is welldefined. In $A K R 97 a, A K R 97 b,{ }^{A K R T 00}$ also the equivalence (2)' $\Leftrightarrow(3)^{\prime}$ has been proved, and obviously (3)' $\Rightarrow(4)^{\prime}$ by taking $v \equiv 0$. So, altogether as in finite dimensions we have:

$$
\text { DLR-version of (1)' } \Leftrightarrow(2)^{\prime} \Leftrightarrow(3)^{\prime} \Rightarrow(4)^{\prime} .
$$

However, as mentioned in the previous subsection the implication " (4)' $\Rightarrow$ (3)'" even under stronger smoothness assumptions on the $U_{\Lambda}$ is a major problem in this infinite dimensional case. In the next section we shall present a result giving a sufficient condition for this to hold.

\section{Remark 2.3.}

(i) Let $\mu$ satisfy (2)' ( $\Leftrightarrow(3)^{\prime} \Leftrightarrow$ DLR-version of (1)'). In this infinite dimensional case ( as in finite dimensions, cf. Remark 2.2) again even the mere existence of $\left(T_{t}^{\mu}\right)_{t \geq 0}=\left(e^{t \hat{L}^{\mu}}\right)_{t \geq 0}$ as a $C_{0}$-semigroup on $L^{1}\left(\mathbb{R}^{\mathbb{Z}^{m}}, \mu\right)$ for a suitable closed extension $L^{\mu}$ of $L$ is not clear in general and only known under quite stringent assumptions. However, if $\left(T_{t}^{\mu}\right)_{t \geq 0}=\left(e^{t \hat{L}^{\mu}}\right)_{t \geq 0}$ exists and the analogue of (2.2) holds for $\mu$, then as in finite dimensions always $L^{*} \mu=0$ by taking $\frac{d}{d t} \uparrow_{t=0}$. So, "invariance" implies "infinitesimally invariance", but the converse is unlike in finite dimensions in fact known to be wrong in general (cf. Chap. 5b in Ebe99 for counter examples). Concerning the question whether $(4)^{\prime} \Rightarrow(3)^{\prime}$, our result in the next section is therefore more general than just stating it for invariant measures, since we prove it for a larger class. In particular, it generalizes the classical well-known 
results in e.g. ${ }^{H S 81}$, Fris2 since it holds also for infinite products of manifolds (cf. ${ }^{B R W 02}$ ).

(ii) To be precise we mention that in (2)', (3)', (4)' above one has to assume, in addition, each time that $Z_{i} \in L^{2}\left(\mathbb{R}^{\mathbb{Z}^{m}}, \mu\right) \forall i \in \mathbb{Z}^{m}$, in order to have that $\left(L_{Z}, \mathcal{F} C_{b}^{2}\right)$ is an operator on $L^{2}\left(\mathbb{R}^{\mathbb{Z}^{m}}, \mu\right)$ and that the Gibbs measure in (1)' defined through the DLR-equations is tempered in a suitable sense. We suppressed this point above since in applications, the square integrability of $Z_{i}$ is automatic (cf. Section 4 below on applications).

\section{Infinitesimally invariance implies Gibbsian}

Consider the situation described in Subsection 2.2, so $U_{\Lambda}, Z=\left(Z_{i}\right)_{i \in \mathbb{Z}^{m}}$, $L_{Z}$ are as defined there.

Assumptions on the potentials $U_{\Lambda}$ : Let $U_{\Lambda}, \Lambda \subset \mathbb{Z}^{m},|\Lambda|<\infty$, satisfy assumption (2.5) for some (fixed) $R>0$, and in addition:

$$
\begin{aligned}
\text { For } E_{k}:=\sum_{\Lambda: \Lambda \subset \Lambda_{k}} U_{\Lambda}, \quad k \in \mathbb{N}, \\
e^{-E_{k}} \in L^{1}\left(\mathbb{R}^{\Lambda_{k}}, \prod_{i \in \Lambda_{k}} d x_{i}\right),
\end{aligned}
$$

where

$$
\Lambda_{k}:=\left\{s \in \mathbb{Z}^{m}\left|\max _{1 \leq i \leq m}\right| s_{i} \mid \leq k R\right\}
$$

Remark 3.1. We note that obviously for $k \in \mathbb{N}$

$$
E_{k}(x)=E_{k}\left(x_{\Lambda_{k}}\right), \quad x \in \mathbb{R}^{\mathbb{Z}^{m}},
$$

and for $i \in \mathbb{Z}^{m}$

$$
Z_{i}(x)=Z_{i}\left(x_{i+\Lambda_{1}}\right), \quad x \in \mathbb{R}^{\mathbb{Z}^{m}},
$$

so, if $i \in \Lambda_{k}$,

$$
Z_{i}(x)=Z_{i}\left(x_{\Lambda_{k+1}}\right), \quad x \in \mathbb{R}^{\mathbb{Z}^{m}} .
$$

Now we can formulate one of the main results from $B R W 02$.

Theorem 3.1. Let $\mu$ be a probability measure on $\mathbb{R}^{\mathbb{Z}^{m}}$ such that 
(i) $Z_{i} \in L^{2}\left(\mathbb{R}^{\mathbb{Z}^{m}}, \mu\right) \quad \forall i \in \mathbb{Z}^{m}$,

(ii) $L_{Z}^{*} \mu=0$, i.e. $\mu$ is $L_{Z}$-infinitesimally invariant,

(iii) $\partial_{i} E_{k} \in L^{2}\left(\mathbb{R}^{\mathbb{Z}^{m}}, \mu\right) \quad \forall i \in \Lambda_{k}, k \in \mathbb{N}$.

Set for $k \in \mathbb{N}$

$$
D_{k}^{\mu}:=\sum_{i \in \Lambda_{k} \backslash \Lambda_{k-1}} \int\left|\mathbb{E}^{\mu}\left[Z_{i} \mid \sigma\left(\Lambda_{k}\right)\right]-\partial_{i} E_{k}\right|^{2} d \mu,
$$

where $\mathbb{E}^{\mu}\left[\cdot \mid \sigma\left(\Lambda_{k}\right)\right]$ denotes conditional expectation of $\mu$ with respect to the $\sigma$-algebra $\sigma\left(\Lambda_{k}\right)$ generated by the map $x \mapsto x_{\Lambda_{k}}, x \in \mathbb{R}^{\mathbb{Z}^{m}}$. If there exist $c_{k} \in\left[D_{k}^{\mu}, \infty\right) \cap(0, \infty)$ such that

$$
\sum_{k=1}^{\infty} \frac{1}{c_{k}+c_{k+1}}=\infty
$$

then $\mu$ is $L_{Z}$-symmetrizing, i.e. Gibbsian.

Instead of giving an account of the proof of Theorem 3.1 we refer to ${ }^{B R W 02}$ and shall rather discuss an application in the next section. We only mention here that the crucial quantities $D_{k}^{\mu}, k \in \mathbb{N}$, in (3.2) exactly capture how strongly the PDE's in (3)' and (4)' are coupled with respect to the one dimensional coordinates of $x=\left(x_{i}\right)_{i \in \mathbb{Z}^{m}}$ or "how much" $\mu$ differs from a product measure on $\mathbb{R}^{\mathbb{Z}^{m}}$. This will become, particularly, clear in the applications below. (3.3) just says that $D_{k}^{\mu}$ should not grow too fast with $k$.

\section{Application}

In this section we shall apply the above, in particular, Theorem 3.1, to a well-studied model from statistical mechanics (cf. e.g. ${ }^{B H K 82}$ and the references therein). This is a lattice system over $\mathbb{Z}^{m}$ with a two-body interaction of finite range $R>0$, i.e. in the frame from the previous section we have for finite $\Lambda \subset \mathbb{Z}^{m}$ :

$$
U_{\Lambda} \equiv 0, \quad \text { unless } \operatorname{diam} \Lambda \leq R \text { and }|\Lambda| \leq 2 .
$$

If $\Lambda=\{i\}$ we set

$$
V_{i}:=U_{\{i\}},
$$

and if $\Lambda=\{i, j\}, i \neq j$, we set

$$
W_{i, j}:=U_{\{i, j\}} .
$$


So, in particular, $W_{i, j}=W_{j, i}$.

Assumptions on $V_{i}, W_{i, j}$ :

$$
V_{i} \in C^{1}(\mathbb{R}), \quad W_{i, j} \in C^{1}\left(\mathbb{R}^{2}\right) .
$$

There exist $K, K_{0} \in(0, \infty), p \in(m, \infty)$ such that $K>12 K_{0}(1+R)^{2+p}$, and $C \in(0, \infty), \alpha \in[2, \infty)$, such that for all $i, j \in \mathbb{Z}^{m}$ and all $s, t \in \mathbb{R}$

$$
\begin{gathered}
\left|W_{i, j}(s, t)\right| \leq K_{0}\left(1+|s|^{\alpha}+|t|^{\alpha}\right), \\
\left|\partial_{1} W_{i, j}(s, t)\right| \leq K_{0}\left(1+|s|^{\alpha-1}+|t|^{\alpha-1}\right), \\
s V_{i}^{\prime}(s) \geq K|s|^{\alpha}-C .
\end{gathered}
$$

It can be easily shown that (4.2), (4.3) imply conditions (2.5) and (3.1).

Let us first calculate the corresponding $Z_{i}$ and $\partial_{i} E_{k}$. We have for $i \in$ $\mathbb{Z}^{m}, k \in \mathbb{N}$, in this situation

$$
\begin{gathered}
Z_{i}(x)=-V_{i}^{\prime}\left(x_{i}\right)-\sum_{\substack{j \in \mathbb{Z}^{m} \\
|i-j| \leq R}} \partial_{1} W_{i, j}\left(x_{i}, x_{j}\right) \\
\partial_{i} E_{k}(x)=-V_{i}^{\prime}\left(x_{i}\right)-\sum_{\substack{j \in \Lambda_{k} \\
|i-j| \leq R}} \partial_{1} W_{i, j}\left(x_{i}, x_{j}\right), \quad \text { if } i \in \Lambda_{k} .
\end{gathered}
$$

By Examples 6.12 and $4.6^{B R 01}$ there exist probability measures $\mu$ on $\mathbb{R}^{\mathbb{Z}^{m}}$ such that the following properties hold:

(A) $L_{Z}^{*} \mu=0$.

(B) ("temperedness of $\mu$ ")

$$
\mu\left(\left\{x=\left.\left(x_{i}\right)_{i \in \mathbb{Z}^{m}} \in \mathbb{R}^{\mathbb{Z}^{m}}\left|\sum_{i \in \mathbb{Z}^{m}} \frac{1}{\max \left(|i|^{p}, 1\right)}\right| x_{i}\right|^{\alpha}<1\right\}\right)=1 .
$$

Furthermore, for all such $\mu$ and every $r \in(0, \infty)$ there exists $M_{r} \in(0, \infty)$ such that

$$
\int_{\mathbb{R}^{\mathbb{Z}^{m}}}\left|x_{i}\right|^{r} \mu(d x) \leq M_{r} \quad \forall i \in \mathbb{Z}^{m},
$$

in particular (by $(4.2),(4.3))$

$$
Z_{i}, \partial_{i} E_{k} \in L^{2}\left(\mathbb{R}^{\mathbb{Z}^{m}}, \mu\right)
$$

for $i \in \mathbb{Z}^{m}$ and $k \in \mathbb{N}$ is such that $i \in \Lambda_{k}$. 
Now let us calculate the crucial quantities $D_{k}^{\mu}, k \in \mathbb{N}$, from the conditions of Theorem 3.1. Fix $k \in \mathbb{N}$ and $i \in \Lambda_{k}$, then since $\partial_{i} E_{k}$ is $\sigma\left(\Lambda_{k}\right)$ measurable we can use Jensen's inequality to obtain

$$
\begin{aligned}
\int\left|\mathbb{E}^{\mu}\left[Z_{i} \mid \sigma\left(\Lambda_{k}\right)\right]-\partial_{i} E_{k}\right|^{2} d \mu \\
\leq \int\left|Z_{i}-\partial_{i} E_{k}\right|^{2} d \mu \\
\quad=\int\left|\sum_{\substack{j \in \mathbb{Z}^{m} \backslash \Lambda_{k} \\
|i-j| \leq R}} \partial_{1} W_{i, j}\left(x_{i}, x_{j}\right)\right|^{2} \mu(d x),
\end{aligned}
$$

where we used (4.4), (4.5) in the last step. By (4.3) and Hölder's inequality the latter is bounded by

$$
\begin{gathered}
3(2 R+1)^{m} K_{0}^{2} \sum_{\substack{j \in \mathbb{Z}^{m} \backslash \Lambda_{k} \\
|i-j| \leq R}} \int\left(1+\left|x_{i}\right|^{2 \alpha-2}+\left|x_{j}\right|^{2 \alpha-2}\right) \mu(d x) \\
\leq 3(2 R+1)^{2 m} K_{0}^{2}\left(1+2 M_{2 \alpha-2}\right)=: C\left(R, m, K_{0}, \alpha\right)
\end{gathered}
$$

where we used (4.7) in the second step. So, for any such $\mu$ as above and $k \in \mathbb{N}$

$$
D_{k}^{\mu} \leq C\left(R, m, K_{0}, \alpha\right)\left|\Lambda_{k} \backslash \Lambda_{k-1}\right|=: c_{k} .
$$

Note that the latter is of order $k^{m-1}$, so

$$
\sum_{k=1}^{\infty} \frac{1}{c_{k}+c_{k+1}} \approx \sum_{k=1}^{\infty} \frac{1}{k^{m-1}+(k+1)^{m-1}}
$$

where " $\approx$ " means "equal up to a constant". So, Theorem 3.1 applies if and only if $m \leq 2$. Hence we have

Corollary 4.1. For the one- and two-dimensional lattices $\mathbb{Z}^{1}$ and $\mathbb{Z}^{2}$ for the above model we have:

$$
\text { infinitesimal invariance implies Gibbsian. }
$$

\section{Concluding Remark 4.2.}

(i) Let $\mathfrak{M}$ denote the set of all probability measures on $\mathbb{R}^{\mathbb{Z}^{m}}$ satisfying conditions (A), (B) above. By similar techniques one can prove (cf. $B R W 02)$ : if one $\mu \in \mathfrak{M}$ satisfies the logarithmic Sobolev inequality, then $\# \mathfrak{M}=1$, i.e. we have uniqueness in this case. This extends results known for compact spin spaces (i.e. lattices of type $\prod_{i \in \mathbb{Z}^{m}} M_{i}$, 
with $M_{i}$ compact manifolds, cf. ${ }^{S Z 92 a}, S Z 92 c, S Z 92 b, S Z 95, Z$ Zeg92, Zeg96) to the non-compact case (see also ${ }^{Y o s 01}$ for a particular case with $M_{i}=\mathbb{R}$ as above).

(ii) There are examples on $\mathbb{R}^{\mathbb{Z}^{m}}$ where "infinitesimal invariance $\Rightarrow$ Gibbsian" for all lattice dimensions $m$. We refer to ${ }^{B R W 02}$ for details.

\section{Acknowledgement}

It is a pleasure for the author to thank Professor H. Heyer for a very pleasant and stimulating conference in Tübingen and for the financial support through the DFG. Financial support of the BiBoS-Research Centre and the German Science Foundation (DFG) through the DFG-Research Group "Spectral Analysis, Asymptotic Distributions and Stochastic Dynamics" is also gratefully acknowledged.

\section{References}

ABR99. S. Albeverio, V. I. Bogachev, and M. Röckner, On uniqueness of invariant measures for finite and infinite dimensional diffusions, Comm. Pure Appl. Math. 52 (1999), 325-362.

AKPR04. S. Albeverio, Y. G. Kondratiev, T. Pasurek, and M. Röckner, Euclidean Gibbs measures on loop lattices: existence and a priori estimates, Ann. Prob. 32 (2004), no. 1A, 153-190.

AKR97a. L Ergodicity of $L^{2}$-semigroups and extremality of Gibbs states, J. Funct. Anal. 144 (1997), 394-423.

AKR97b. S. Albeverio, Y. G. Kondratiev, and M. Röckner, Ergodicity for the stochastic dynamics of quasi-invariant measures with applications to Gibbs states, J. Funct. Anal. 149 (1997), 415-469.

AKRT00. S. Albeverio, Y. G. Kondratiev, M. Röckner, and T. V. Tsikalenko, A-priori estimates on symmetrizing measures and their applications to Gibbs states, J. Funct. Anal. 171 (2000), 366-400.

ARZ93. S. Albeverio, M. Röckner, and T.-S. Zhang, Markov uniqueness for a class of infinite dimensional Dirichlet operators, Stochastic Processes and Optimal Control (H. J. Engelbert et al., eds.), Stochastic Monographs, vol. 7, Gordon \& Breach, 1993, pp. 1-26.

BHK82. J. Bellissard and R. Høegh-Krohn, Compactness and the maximal Gibbs states for random Gibbs fields on a lattice, Comm. Math. Phys. 84 (1982), 297-327.

BKR97. V. I. Bogachev, N. V. Krylov, and M. Röckner, Elliptic regularity and essential self-adjointness of Dirichlet operators on $\mathbb{R}^{d}$, Ann. Scuola Norm. Sup. Pisa Cl. Sci., Serie IV XXIV (1997), no. 3, 451-461.

BR95. V. I. Bogachev and M. Röckner, Regularity of invariant measures on finite and infinite dimensional spaces and applications, J. Funct. Anal. 133 (1995), 168-223. 
BR01. Elliptic equations for measures on infinite dimensional spaces and applications, Prob. Th. Rel. Fields 120 (2001), 445-496.

BR03. _ On $L^{p}$-uniqueness of symmetric diffusion operators on Riemannian manifolds, Matem. Sbornik. 194(7) (2003), 969-978.

BRS00. V. I. Bogachev, M. Röckner, and W. Stannat, Uniqueness of invariant measures and essential m-dissipativity for diffusion operators on $L^{1}$, Infinite dimensional Stochastic Analysis (P. Clément et al., eds.), Royal Netherlands Academy of Arts and Sciences, Amsterdam, 2000, pp. 39-54.

BRS02. Uniqueness of solutions of elliptic equations and uniqueness of invariant measures of diffusions, Matem. Sbornik. 193:7 (2002), 3-36, (Russian version), 945-976 (English version).

BRW01. V. I. Bogachev, M. Röckner, and F.-Y. Wang, Elliptic equations for invariant measures on finite and infinite dimensional manifolds, J. Math. Pures Appl. 80 (2001), 177-221.

BRW02. L Invariance implies Gibbsian: some new results, BiBoSPreprint 02-12-106 (2002), to appear in Comm. Math. Phys., 18 pp.

Chu83. L. O. Chung, Existence of harmonic $L^{1}$-functions in complete Riemannian manifolds, Proc. Amer. Math. Soc. 88 (1983), 531-532.

Ebe99. A. Eberle, Uniqueness and non-uniqueness of semigroups generated by singular diffusion operators, Lecture Notes in Math., vol. 1718, Springer, Berlin, 1999.

Fri82. J. Fritz, Stationary measures of stochastic gradient systems, infinite lattice models, Z. Wahr. verw. Geb. 59 (1982), 479-490.

Geo88. H.-O. Georgii, Gibbs measures and phase transitions, de Gruyter, 1988.

HS81. R. Holley and D. W. Stroock, Diffusions on an infinite dimensional torus, J. Funct. Anal. 42 (1981), 29-63.

LS84. $\quad \mathrm{P}$. Li and R. Schoen, $L^{p}$ and mean value properties of subharmonic functions on Riemannian manifolds, Acta Mathematica 153 (1984), 279-301.

Sta99. W. Stannat, (Nonsymmetric) Dirichlet operators on $L^{1}$ : Existence, uniqueness and associated Markov processes, Ann. Scuola Norm. Sup. Pisa Cl. Sci., Serie IV 28 (1999), 99-140.

SZ92a. D. W. Stroock and B. Zegarlinski, The equivalence of the logarithmic Sobolev inequality and the Dobrushin-Shlosman mixing condition, Comm. Math. Phys. 144 (1992), no. 2, 303-323.

SZ92b. ㄴ The logarithmic Sobolev inequality for continuous spin systems on a lattice, J. Funct. Anal. 104 (1992), no. 2, 299-326.

SZ92c. 는 The logarithmic Sobolev inequality for discrete spin systems on a lattice, Comm. Math. Phys. 149 (1992), no. 1, 175-193.

SZ95. On the ergodic properties of Glauber dynamics, J. Stat. Phys. 81 (1995), no. 5-6, 1007-1019.

Yos01. N. Yoshida, The equivalence of the log-Sobolev inequality and a mixing contition for unbounded spin systems on the lattice, Ann. Inst. H. Poincaré Probab. Statist. 37 (2001), no. 2, 223-243.

Zeg92. B. Zegarlinski, Dobrushin uniqueness theorem and logarithmic Sobolev 
inequalities, J. Funct. Anal. 105 (1992), no. 1, 77-111.

Zeg96. ㄴ. The strong decay to equilibrium for the stochastic dynamics of unbounded spin systems on a lattice, Comm. Math. Phys. 175 (1996), no. 2, 401-432. 